\title{
A MODIFICATION OF GROTHENDIECK'S SPECTRAL SEQUENCE
}

\author{
EDGAR ENOCHS
}

\section{Introduction}

Let $C, C^{\prime}$ and $C^{\prime \prime}$ be abelian categories where $C$ and $C^{\prime}$ have enough injectives and let $F: C \rightarrow C^{\prime}, G: C^{\prime} \rightarrow C^{\prime \prime}$ be additive covariant functors. Then for an object $X$ of $C$, let $C(X)$ be the complex associated with an injective resolution of $X$. Grothendieck gets a first quadrant spectral sequence by taking an injective resolution of the complex $F(C(X))$ and applying $G$ to the associated double complex. Under certain hypotheses one gets a spectral sequence

$$
E_{2}^{p q}=R^{p} G\left(R^{q} F(X)\right) \Rightarrow R^{n}(G F)(X) .
$$

If we modify this procedure by replacing $C(X)$ with a projective resolution of $X$ and then proceed as above, we get a second quadrant spectral sequence. Using these spectral sequences, a variety of known results can be proved and sharpened.

In the first applications $C=C^{\prime}$ and $F=\mathrm{id}_{c}$, so initially to simplify notation make this assumption (Grothendieck's spectral sequence becomes trivial in this case). Some applications will require slight changes in these hypotheses, but it will then be easy to see how to modify the proofs.

\section{$\S 1$. The spectral sequence}

In this paper we adopt the convention that the derived functors $R^{q} T$ $=0$ when $q<0$ and similarly $L_{p} T=0$ for $p<0$. Also Betti and Bass numbers with strictly negative subscripts will be taken to be 0 .

Proposition 1.1. Let $T: C \rightarrow C^{\prime}$ be an additive functor where $C$ and $C^{\prime}$ are abelian categories and $C$ has enough injectives and projectives. Then for an object $X$ of $C$ there is a second quadrant spectral sequence

Received February 12, 1987. 


$$
E_{2}^{-p, q}=L_{p}\left(R^{q} T\right)(X) \Rightarrow R^{n} T(X)
$$

if either a) hd $(X)<\infty$ or b) inj $\operatorname{dim} T<\infty$ (i.e. $R^{q} T=0$ for $q$ sufficiently large).

Proof. We consider a commutative diagram

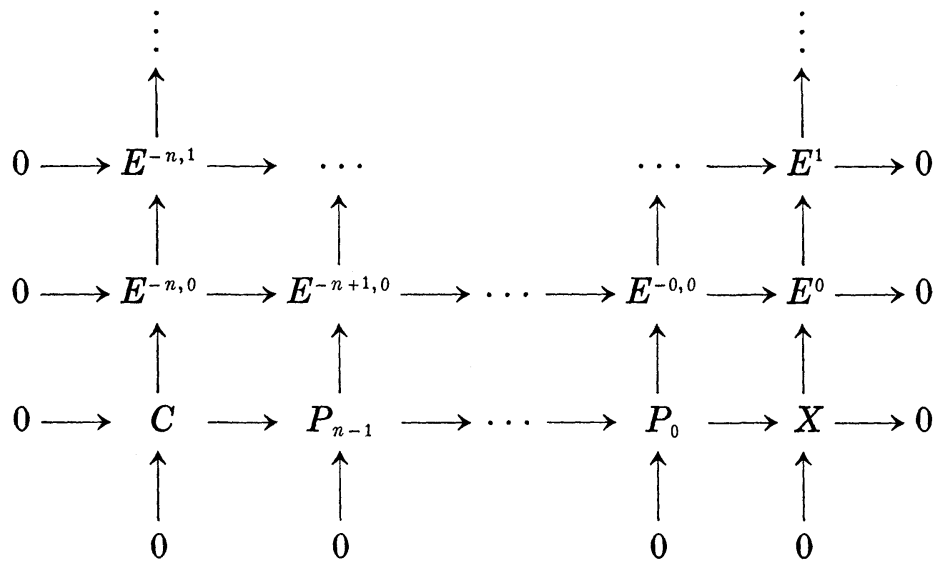

with exact rows and columns, where each $P_{i}$ is projective, and where each $E^{i, j}$ and $E^{j}$ is injective. If a) holds, assume $C$ is projective and write $C=P_{n}$. Then using the first filtration on the double complex $\left(T\left(E^{i, j}\right)\right)$ we get

$$
E_{1}^{-p, q}=\left(R^{q} T\right)\left(P_{p}\right) \text { and so } E_{2}^{-p, q}=L_{p}\left(R^{q} T\right)(X) \text {. }
$$

Using the second filtration we see that since we can peel off the injectives on the left, the spectral sequence collapses and we get that the homology of the double complex is $R^{n}(T(X))$.

Now assume b). Then for each $n$ we get such a diagram. However we can assume that if $n^{\prime} \geq n$ then the objects $E^{i, j}$ and $P_{i}$ and the maps between them for $i \geq-n+1$ are the same for both diagrams. Now we consider the first filtration and the associated spectral sequences. Without the hypothesis b), for a fixed $p, q$ and $r, E_{r}^{-p, q}$ is independent of $n$ for large $n$. But with the hypothesis b) we see that if we only fix $p$ and $q, E_{r}^{-p, q}$ is independent of $n$ for large $n$ for all $r$. Hence $E_{\infty}^{-p, q}$ can be defined independently of $n$. So we get a spectral sequence by taking the limit (in the obvious sense) of the spectral sequences we get for each $n$. Since each of these sequences converges to $R^{n} T(X)$, so does the limit sequence. 
The idea for this case was suggested by a comment (without proofs) of Grothendieck ([1], pg. 147). We note there is a dual procedure giving rise to a third quadrant spectral sequence.

We make note of a more general version. Assume $C$ has enough projectives and $C^{\prime}$ enough injectives. Let $F: C \rightarrow C^{\prime}, G: C^{\prime} \rightarrow C^{\prime \prime}$ be additive covariant functors such that $\operatorname{proj} \operatorname{dim} F<\infty$ or $\operatorname{inj} \operatorname{dim} G<\infty$. Then there is a spectral sequence

$$
E_{2}^{-p, q}=L_{p} F\left(R^{q} G(X)\right) \Rightarrow \boldsymbol{R}^{n}(F \circ G(P .))
$$

where $P$. arises from a projective resolution of $X$ and $\boldsymbol{R}^{n}(F \circ G(P)$.$) is the$ $n$-th hypercohomology of the complex $F \circ G(P$.). Since all applications assume the more restrictive hypotheses and these make the proofs less cumbersome, we restrict ourselves to this case.

Remark. The spectral sequences in the restricted form coincide with Dold's universal coefficient spectral sequences [7]. However his proofs are different.

\section{§2. Duality}

Let $A$ be a commutative ring and $C=C^{\prime}={ }_{A} M$ (the category of $A$ modules) and let $T: C \rightarrow C^{\prime}$ be additive. Then we have:

Proposition 2.1. If $M$ is an A-module, $T$ commutes with direct sums and either hd $M<\infty$ or $\operatorname{inj} \operatorname{dim} T<\infty$, there is a spectral sequence

$$
E_{2}^{-p, q}=\operatorname{Tor}_{p}\left(R^{q} T(A), M\right) \Rightarrow R^{n} T(M) .
$$

Proof. Using a free resolution of $M$, it's easy to see that the hypothesis on $T$ guarantees that $L_{p} R^{q} T(M) \cong \operatorname{Tor}_{p}\left(R^{q} T(A), M\right)$. We then appeal to Proposition 1.1.

Corollary 1 (duality). If $R^{q} T(A)=0$ for $q \neq n \geq 0$ then there are natural isomorphisms

$$
\operatorname{Tor}_{n-i}\left(R^{n} T(A), M\right) \cong R^{i} T(M) .
$$

Proof. Immediate.

Note that the isomorphisms in the Corollary hold for all $i$, so $\operatorname{Tor}_{n+k}\left(R^{n} T(A), M\right)=0$ for $k>0$, implying that the flat dimension of $R^{n} T(A)$ is at most $n$. If $R^{0} T(M) \neq 0$ for some $M$, then it is $n$. 
If $A$ is noetherian and local, let $T=\Gamma_{\mathfrak{m}}$ (the local cohomology functor) with $\mathfrak{M}$ the maximal ideal of $A$. Then $R^{q} T(M)=H_{\mathfrak{R}}^{q}(M)$.

It's known that $H_{\mathfrak{m}}^{q}(M)=0$ if $q>\operatorname{dim} A=d$, so Proposition 2.1 applies and we get the spectral sequence

$$
\operatorname{Tor}_{p}\left(H_{\mathfrak{M}}^{q}(A), M\right) \Rightarrow H_{\mathfrak{m}}^{n}(M) .
$$

Note that this gives the familiar isomorphism

$$
H_{\Re}^{d}(A) \otimes M \cong H_{\mathfrak{M}}^{d}(M) \quad \text { where } d=\operatorname{dim} A .
$$

If $A$ is Cohen-Macaulay, of dimension $d$, then

$$
H_{\mathfrak{M}}^{q}(A)=0 \quad \text { for } q \neq d .
$$

Corollary 2 (Grothendieck duality). If $A$ is a local Cohen-Macaulay ring of dimension $d$ then there are natural isomorphisms

$$
\operatorname{Tor}_{d-i}\left(H_{\mathfrak{M}}^{d}(A), M\right) \cong H_{\mathfrak{M}}^{i}(M)
$$

for all A-modules $M$ (whether finitely generated or not).

If we take the Matlis dual of both sides of the above we get

$$
H_{\mathfrak{m}}^{i}(M)^{\nu} \cong \operatorname{Tor}_{d-i}\left(H_{\mathfrak{N}}^{d}(A), M\right)^{\nu} \cong \operatorname{Ext}^{d-i}(M, \Omega)
$$

with $\Omega=H_{\mathfrak{m}}^{d}(A)^{\nu}$. If $M$ is finitely generated we get the duality as given in Grothendieck ([2], Theorem 6.7, pg. 96). In this case the flat dimension of $H_{\mathfrak{M}}^{p}(A)$ is $d$ since $\operatorname{Tor}_{d}\left(H_{\mathfrak{m}}^{d}(A), k\right) \cong H_{\mathfrak{m}}^{0}(k)=k \neq 0$.

\section{$\S 3$. Change of rings}

Proposition 4.1. Let $A \rightarrow B$ be a ring homomorphism where $A$ is left noetherian and of finite left global dimension. Then for any left B-module $M\left({ }_{B} M\right.$ for short) we have

$$
\text { inj } \operatorname{dim}_{A} M \leq \operatorname{inj} \operatorname{dim}_{A} B .
$$

Proof. For the proof we use a modification of Proposition 1.1. Let $X=M$ in the diagram of the proof and suppose the bottom row is part of a projective resolution of $M$ as a $B$-module. The columns will be injective resolutions of $A$-modules. The hypothesis on $A$ will guarantee we can use b) of Proposition 1.1 when $T=\operatorname{Hom}_{A}(N,-)$ for $N$ a left $A$ module. If in an addition we assume $N$ is finitely generated we get the spectral sequence of Proposition 2.1, which, in this situation, is: 


$$
E_{2}^{-p, q}=\operatorname{Tor}_{p}^{B}\left(\operatorname{Ext}_{A}^{q}(N, B), M\right) \Rightarrow \operatorname{Ext}_{A}^{n}(N, M) .
$$

If inj $\operatorname{dim}_{A} B=s$, then $E_{2}^{-p, q}=0$ for $q>s$ and so $\operatorname{Ext}_{A}^{n}(N, M)=0$ for $n>s$. This completes the proof.

\section{$\S 4$. Growth of Betti numbers}

Letting $M$ be a $B$-module where $B$ is a local ring, we establish linear recurrence inequalities on the Betti numbers of $M$. We note that replacing $B$ and $M$ with their completions the Betti numbers remain unchanged. But a complete local ring $B$ is a quotient of a regular local ring $A$. So assume $A \rightarrow B$ gives $B$ as a quotient of a regular local ring $A$. Let $k=$ $A / M, M$ the maximum ideal and let $\beta_{i}=\beta_{i}\left({ }_{B} M\right), \mu_{i}=\mu_{i}\left({ }_{A} B\right)$. Let $t=$ inj $\operatorname{dim}_{A} B$ and $s=\operatorname{depth} B$ as an $A$-module. Then we have

Proposition 4.1.

$$
\beta_{p} \mu_{t} \leq \sum_{r=2}^{P} \beta_{p-r} \mu_{t+1-r} \quad \text { when } t-p<\operatorname{depth} M
$$

and

$$
\beta_{p} \mu_{r} \leq \sum_{r=2}^{t-s+1} \beta_{p+r} \mu_{s+r-1} \quad \text { when } s-p<\operatorname{depth} M
$$

Proof. Using $T=\operatorname{Hom}_{A}(k,-)$, by a now familiar procedure we get a spectral sequence

$$
E_{2}^{-p, q}=\operatorname{Tor}_{p}^{B}\left(\operatorname{Ext}_{A}^{q}(k, B), M\right) \cong \operatorname{Ext}_{A}^{q}(k, B)^{\beta_{p}}
$$

converging to $\operatorname{Ext}_{A}^{n}(k, M)$.

Note that the dimension of $E_{2}^{-p, q}$ over $k$ is $\beta_{p} \mu_{q}$. We have $E_{2}^{-p, q}=0$ if $q>t$, so no element of $E_{r}^{-p, t}$ for $r \geq 2$ is a boundary. Since $\operatorname{Ext}^{n}(k, M)$ $=0$ if $n<\operatorname{depth} M$, we get $E_{\infty}^{-p, t}=0$ if $t-p<\operatorname{depth} M$. This easily implies that there is an embedding of $E_{2}^{-p, t}$ in $\oplus_{r=2}^{P} E_{2}^{-p+r, t+1-r}$. This gives the first inequality. The argument for the second is similar, but uses the fact that the elements of $E_{2}^{-p, s}$ for $s-p<\operatorname{depth} B$ must eventually become boundaries.

Remark. In case $B=A /(x), x \neq 0$ and $x$ not a unit, then $t=s+1$, $\mu_{s}=1, \mu_{s+1}=1$ and $\mu_{j}=0$ otherwise (by Foxby [9], Corollary 3.2) and the inequalities become

$$
\beta_{p} \leq \beta_{p-2}, \quad \beta_{p} \leq \beta_{p+2}
$$


which implies $\beta_{p}=\beta_{p+2}$ eventually. This is a (very) weak version of Eisenbud's result ([4], Theorem 6.1, pg. 52).

In general it's easy to see (cf. Brualdi [8]) the inequalities guarantee there is a $K>0$ with $\beta_{n} \leq K^{n}$ for all $n$. If, for example, $A=k\left[x_{1}, \cdots, x_{d}\right] /$ $\left(x_{1}, \cdots, x_{d}\right)^{2}, k$ a field and $M=k$, then in fact $\beta_{n}=d^{n}$.

\section{§ 5. Syzygies}

We let $A$ be a local Gorenstein ring of dimension $d$. Given the exact sequence

$$
0 \longrightarrow M \longrightarrow A^{\beta_{s-1}} \longrightarrow \cdots \longrightarrow A_{\beta_{0}} \longrightarrow N \longrightarrow 0
$$

of $A$-modules, $M$ is called an $s$-th syzygy of $N$. If the resolution is assumed minimal, then $M$ is uniquely determined by $N$, but the converse is clearly not true. We do have:

Proposition 5.1. If

$$
\begin{aligned}
& 0 \longrightarrow M \longrightarrow A^{\beta_{s-1}} \longrightarrow \cdots \longrightarrow A^{\beta_{0}} \longrightarrow N \longrightarrow 0 \\
& 0 \longrightarrow M \longrightarrow A^{\beta_{s-1}^{\prime}} \longrightarrow \cdots \longrightarrow A^{\beta_{0}^{\prime}} \longrightarrow N^{\prime} \longrightarrow 0
\end{aligned}
$$

are exact sequences, $s>d$ and both resolutions are minimal, then there exists a commutative diagram

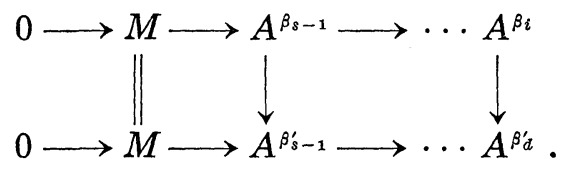

In this case, all vertical maps are isomorphisms.

Proof. We only need to show that there is a commutative diagram

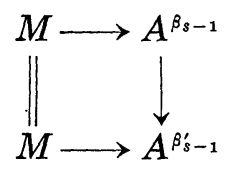

and that $A^{\beta_{s-1}} \rightarrow A^{\beta_{s-1}}$ must be an isomorphism.

Since $\operatorname{Ext}^{s}(N, A)=0$,

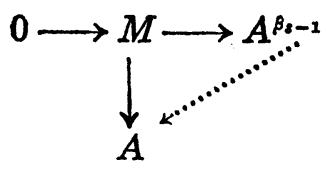


can always be completed, and consequently so can

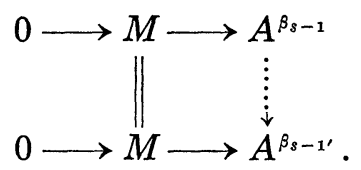

Taking an injective resolution (as in Proposition 1.1) of

$$
0 \longrightarrow M \longrightarrow A^{\beta_{s-1}} \longrightarrow \cdots \longrightarrow A_{\beta_{0}} \longrightarrow N \longrightarrow 0
$$

and letting $T=\operatorname{Hom}(k,-)$, we get a spectral sequence with $E_{1}$

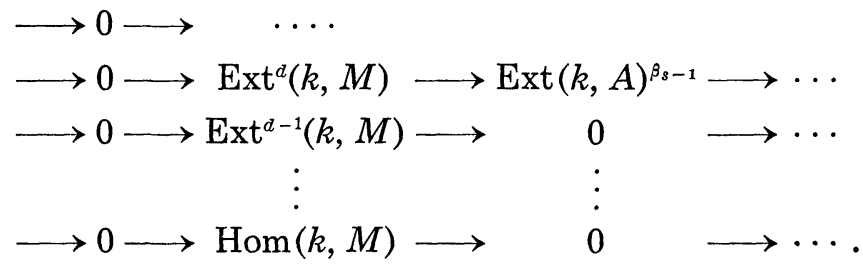

Since $\operatorname{Ext}^{-s+d}(k, N)=0(-s+d<0)$, we see that $\operatorname{Ext}^{d}(k, M) \rightarrow \operatorname{Ext}^{d}(k, A)^{\beta_{s-1}}$ must be an isomorphism.

If we now apply $\operatorname{Ext}^{d}(k,-)$ to the commutative diagram

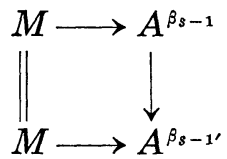

we see that

$$
\operatorname{Ext}^{d}\left(k, A^{\beta_{s-1}}\right)=\operatorname{Ext}^{d}(k, A)^{\beta_{s-1^{\prime}}} \longrightarrow \operatorname{Ext}^{d}(k, A)^{\beta_{s-1}}
$$

must be an isomorphism. Since $\operatorname{Ext}^{d}(k, A) \neq 0$ this gives that $A^{\beta_{s-1}} \rightarrow A^{\beta_{s-1}}$ is an isomorphism.

Remark 1. In the language of Enochs [6], $M \rightarrow A^{\beta_{s-1}}$ is a projective envelope of $M$. We note that any two projective envelopes of $M$ are isomorphic over $M$.

In this case $\left(A^{\beta_{s-1}}\right)^{*} \rightarrow M^{*}\left(M^{*}=\operatorname{Hom}(M, A)\right)$ is a projective cover of $M^{*}$, so any direct sum decomposition of $M^{*}$ gives one of its projective cover. Hence taking duals, we see that any finite direct sum decomposition $M=\oplus M_{i}$ gives one of $A^{\beta_{s-1}}=\oplus P_{i}$ with $M_{i}$ mapped into $P_{i}$. By Eisenbud ([4], Lemma 0.1 (ii), pg. 34), if $s \geq d, M$ has no free summands, so if $M_{i} \neq 0$ then $M_{i} \neq P_{i}$ and so $P_{i} / M_{i}=0$. Hence non-trivial direct 
sum decompositions of $M$ give such decompositions of $A^{\beta_{s-1}} / M$. The converse is also true. This argument quickly gives

CoRollary 1. If $\cdots \longrightarrow A^{\beta_{2}} \stackrel{d_{2}}{\longrightarrow} A^{\beta_{1}} \stackrel{d_{1}}{\longrightarrow} A^{\beta_{0}} \longrightarrow M \longrightarrow 0$ is a minimal projective resolution and $M_{s}=\operatorname{Ker}\left(d_{s-1}\right)$ for any $s \geq 1$, then if $s, t \geq d, M_{s}$ is the direct sum of $k$ indecomposable non-zero submodules, then so is $M_{i}$.

Using the terminology of Eisenbud [4] we also have

CoROLlary 2. If the minimal projective resolution

$$
\cdots \longrightarrow F_{2} \stackrel{d_{2}}{\longrightarrow} F_{1} \stackrel{d_{1}}{\longrightarrow} F_{0} \longrightarrow N \longrightarrow 0
$$

is periodic then it becomes periodic after $d+1$ steps.

Proof. By hypothesis we have that if $i$ is sufficiently large for some $M=\operatorname{Ker}\left(d_{i}\right) \cong \operatorname{Ker}\left(d_{i+n k}\right)$ for some $k>0$ and all $n \geq 0$. Assume $i \geq d$ and apply the fact that $M \rightarrow F_{i+n k}$ and $M \rightarrow F_{i}$ are projective envelopes so lead to an isomorphism $F_{i+n k} \rightarrow F_{i}$ over $M$. This in turn gives an isomorphism $F_{i-1+n k} / M \rightarrow F_{i} / M$. Repeating the argument we get an isomorphism $F_{i-1+n k} \rightarrow F_{i-1}$. In this way we complete the proof.

We note Eisenbud proves this result (and more) in case $A$ is a complete intersection (see Theorem 4.1, pg. 47 of [4]). Ramras has a closely related result ([5], Proposition 1.4, pg. 196), but his concern was with $N$ whose Betti numbers are eventually constant or go to infinity.

Remark 2. Proposition 5.1 can be regarded as a commutative, higher dimensional generalization of Theorem 6.31 of Curtis and Reiner [12] where $A$ is taken to be quasi-Frobenius (so Gorenstein of dimension 0 when commutative). As they note, results of Alperin and Janusz in [11] on the periodicity of projective resolution follow from their theorem.

\section{REFERENCES}

[1] A. Grothendieck, Sur quelques points d'algèbre homologique, Tôhoku Math. J., Series 2, 9, No. 2 (1957), 119-221.

[2] - Local Cohomology (notes by R. Hartshorne).

[ 3 ] P. Roberts, Homological invariants of modules over commutative rings, Séminaire de mathématiques supérieures, L'Université de Montréal (1980).

[ 4 ] D. Eisenbud, Complete intersections, Trans. Amer. Math. Soc., 260 (1980), 35-64.

[5] M. Ramras, Sequences of Betti numbers, J. Algebra, 66 (1980), 193-204.

[ 6 ] E. Enochs, Injective and flat covers, envelopes and resolvents, Israel J. Math., 39 (8) (1981), 189-209. 
[ 7 ] A. Dold, Universelle Koeffizienten, Math. Z., 80 (1962), 63-88.

[8] R. Brualdi, Introductory Combinatorics, North Holland, New York, 1977.

[9] H. Foxby, On the $\mu^{i}$ in a minimal injective resolution, Math. Scand., 29 (1971), 175-186.

[10] H. Cartan and S. Eilenberg, Homological Algebra, Princeton Math. Ser. 19, Princeton, 1956.

[11] J. L. Alperin and G. J. Janusz, Resolutions and periodicity, Proc. Amer. Math. Soc., 37 (1973), 403-406.

[12] C. W. Curtis and I. Reiner, Methods of Representation Theory, Vol. I, John Wiley and Sons, New York, 1981.

Department of Mathematics

University of Kentucky

Lexington, Kentucky 40506-6027

U.S.A. 